\title{
Intent To Treat
}

National Cancer Institute

\section{Source}

National Cancer Institute. Intent To Treat. NCI Thesaurus. Code C54398.

The use of a particular medical device, medication or treatment to treat a pre-existing disease or condition. 